\title{
A Truncated Species of Apolipoprotein B (B67) in a Kindred with Familial Hypobetalipoproteinemia
}

\author{
Francine K. Welty, ${ }^{*}$ Susan T. Hubl, Vincenzo R. Pierotti, and Stephen G. Young \\ ${ }^{*}$ Cardiology Division, Institute for the Prevention of Heart Disease, New England Deaconess Hospital, \\ Harvard Medical School, Boston, Massachusetts 02215; and Gladstone Foundation Laboratories for Cardiovascular Disease, \\ Cardiovascular Research Institute, Department of Medicine, University of California, San Francisco, San Francisco, \\ California 94140-0608
}

\begin{abstract}
We describe a kindred in which the prcband and 6 of his 12 children have hypobetalipoproteinemia. The plasma lipoproteins of the affected subjects contained a unique species of apolipoprotein (apo) B, apo B67, in addition to the normal species, apo $B 100$ and apo B48. The size of apo B67 and immunochemical studies with a panel of apo B-specific antibodies indicated that apo B67 was a truncated species of apo $B$ that contained approximately the amino-terminal 3,000-3,100 amino acids of apo $B 100$. Sequencing of genomic apo $B$ clones revealed that affected family members were heterozygous for a mutant apo $B$ allele containing a single nucleotide deletion in exon 26 (cDNA nucleotide 9327). This frameshift mutation is predicted to result in the synthesis of a truncated apo $B$ containing 3,040 amino acids. Apo $B 67$ is present in low levels in the plasma but is easily detectable within the very low density lipoprotein and low density lipoprotein fractions. Examination of the proband's immediate family revealed seven normolipidemic subjects and seven subjects with hypobetalipoproteinemia. In the affected subjects, the mean total and low density lipoprotein cholesterol levels were 120 and $42 \mathrm{mg} / \mathrm{dl}$, respectively. A significantly higher mean high density lipoprotein cholesterol level was found in the affected subjects (75 vs. $55 \mathrm{mg} / \mathrm{dl}$ ). We hypothesize that the elevated high density lipoprotein cholesterol levels in subjects heterozygous for the apo B67 mutation may be metabolically linked to the low levels of apo B-containing lipoproteins in their plasma. (J. Clin. Invest. 1991. 87:1748-1754.). Key words: apolipoprotein B • cholesterol • hyperalphalipoproteinemia $\bullet$ hypolipidemia $\bullet$ polymerase chain reaction
\end{abstract}

\section{Introduction}

Within the past 3 years, investigators from several laboratories have demonstrated that familial hypobetalipoproteinemia can

\footnotetext{
A portion of the data in this article was presented at the 1989 and 1990 Scientific Sessions of the American Heart Association.

Address reprint requests to either Dr. Welty, Section of Cardiology, New England Deaconess Hospital, 185 Pilgrim Road, Boston, MA 02215; or Dr. Young, Gladsone Foundation Laboratories for Cardiovascular Disease, P.O. Box 40608, San Francisco, CA 94140-0608.

Received for publication 2 October 1990 and in revised form 28 December 1990.
}

J. Clin. Invest.

(C) The American Society for Clinical Investigation, Inc.

$0021-9738 / 91 / 05 / 1748 / 07 \$ 2.00$

Volume 87, May 1991, 1748-1754 be caused by a variety of apolipoprotein (apo) B gene mutations that prevent the translation of a full-length apo B100 (1). Several of these apo B gene mutations result in the synthesis of a truncated species of apo B that can be detected within the plasma lipoproteins (1). Individuals homozygous for familial hypobetalipoproteinemia typically have extremely low low density lipoprotein (LDL) cholesterol levels, usually $<5 \mathrm{mg} / \mathrm{dl}$ (2). Because apo B synthesis is required for intestinal absorption of dietary fats, these homozygotes may have clinical symptoms related to malabsorption of fats and fat-soluble vitamins. Although the absorption of dietary fats in carefully documented familial hypobetalipoproteinemia heterozygotes has not yet been rigorously examined, it is rare for these heterozygotes to report any symptoms of fat malabsorption (2). Heterozygotes typically have total plasma cholesterol levels in the range of $90-150 \mathrm{mg} / \mathrm{dl}$, with $\mathrm{LDL}$ cholesterol levels in the range of $25-50 \mathrm{mg} / \mathrm{dl}(1)$. Because of their low cholesterol levels, heterozygotes may actually be protected from the development of atherosclerotic coronary artery disease (3). Glueck and co-workers (4) have reported evidence suggesting that subjects with familial hypobetalipoproteinemia may live longer than normocholesterolemic subjects.

Recently, in screening a rural midwestern population, one of the authors (Dr. Welty) identified an adult male subject who had very low cholesterol levels even though he consumed a diet rich in cholesterol and saturated fats (5). Examination of the proband's 12 children revealed that the phenotype of hypobetalipoproteinemia was inherited in a simple Mendelian fashion. In this article, we show that the plasma of hypocholesterolemic family members contained a unique truncated species of apo B, apo B67. Apo B67 was easily detectable within the lipoproteins of the very low density lipoprotein (VLDL) and LDL fractions. Apo B67 is synthesized as a result of a single nucleotide deletion in the apo $B$ gene. The report of this kindred and their unique apo $B$ gene mutation is informative for several reasons. Examination of the lipoproteins of affected family members provides important support for the hypothesis that the length of apo $B$ is an important determinant of the buoyant density of apo B-containing lipoproteins. Based on our prior studies, we had hypothesized that the longer the apo B, the more buoyant the apo B-containing lipoproteins, presumably because longer apo B's contain more lipid-binding regions. The apo B67 mutation supports this hypothesis, in that we found that apo B67-containing lipoproteins are more buoyant than apo B31- and apo B37-containing lipoproteins, yet slightly more dense than apo B86-containing lipoproteins. This kindred is also interesting because affected family members have elevated high density lipoprotein (HDL) cholesterol levels, as compared with the values in population controls and unaffected family members. We hypothesize that low lev- 
els of apo B-containing lipoproteins in affected family members may be metabolically linked to elevated HDL cholesterol levels.

\section{Methods}

Human subjects. Cholesterol measurements were obtained in 126 subjects in a rural midwestern town. Many of these subjects were members of farm families who consume a diet rich in saturated fats and cholesterol. One subject, the proband of the kindred described below, had a total plasma cholesterol concentration of $151 \mathrm{mg} / \mathrm{dl}$. Additional blood samples were taken from the proband, as well as from his 12 offspring. The presence of angina pectoris in the proband and his family was assessed by the Rose questionnaire (6). A 12-lead electrocardiogram was performed on each family member.

Analysis of lipid levels and blood chemistry. Blood samples were obtained after the subjects had fasted for $14 \mathrm{~h}$. The blood was collected into tubes containing EDTA ( $1 \mathrm{mg}$ of EDTA $/ \mathrm{ml}$ of blood), and the plasma was immediately separated by centrifugation. Total cholesterol, LDL cholesterol, HDL cholesterol, and triglycerides were measured according to Lipid Research Clinic techniques (7) in a laboratory participating in the standardization program of the Centers for Disease Control, Atlanta, GA. Serum thyroxin levels, vitamin A, and vitamin E levels were determined using standard techniques. The concentrations of apo A-I and apo B in plasma were determined by radioimmunoassays.

Analysis of diet. The diet of the proband and his family was analyzed by a detailed questionnaire. Each individual provided a complete written record of everything they consumed during a 3-d period. No recall was involved. The total caloric intake per day, the percent carbohydrate, protein, saturated fat, monounsaturated fat and polyunsaturated fat, and cholesterol were determined using the University of Minnesota dietary analysis computer program (8). The height and weight of each subject were recorded, and the ratio of the subject's weight divided by ideal body weight was calculated (9). The body mass index [weight in kilograms/(height in meters) ${ }^{2}$ ] was also calculated (9).

Isolation and characterization of lipoproteins. The VLDL $(d$ $<1.006 \mathrm{~g} / \mathrm{ml}$ ), intermediate density lipoprotein (IDL) ${ }^{1}(1.006-1.025$ $\mathrm{g} / \mathrm{ml}), \mathrm{LDL}(1.025-1.063 \mathrm{~g} / \mathrm{ml})$, and HDL $(1.063-1.21 \mathrm{~g} / \mathrm{ml})$ fractions were isolated from fresh plasma by ultracentrifugation $(10)$. The protein content of the lipoproteins was determined by a Lowry protein assay in which bovine serum albumin was the standard (11). The apolipoprotein content of each lipoprotein fraction was assessed by $3-12 \%$ gradient sodium dodecyl sulfate-polyacrylamide gel electrophoresis (SDS-PAGE) on gels stained with 0.1\% Coomassie Brilliant Blue R-250 or with silver. In some cases, the apolipoproteins separated by SDSPAGE were electrophoretically transferred to nitrocellulose membranes for Western blotting $(12,13)$. For Western blots, we used a variety of previously characterized apo B-specific antibodies $(12,14$ 18). The size of lipoprotein particles within the LDL and HDL fractions was assessed by nondenaturing gradient polyacrylamide gels, as previously described $(15,19,20)$. In some cases, the lipoproteins separated according to size by the nondenaturing gels were electrophoretically transferred to nitrocellulose membranes for Western blots (15).

Enzymatic amplification of DNA and DNA sequencing. The SDSpolyacrylamide gels revealed a unique truncated apo B species, apo B67, in the lipoproteins of affected family members. The size of the protein, as well as its reactivity with apo B-specific antibodies suggested that its carboxy terminus must be in the vicinity of apo B 100 amino acid residues 3030-3050 (see Results). To determine the precise mutation in the apo B gene responsible for apo B67, a 1,126-base pair

1. Abbreviation used in this paper: IDL, intermediate density lipoprotein. segment of exon 26 of the apo B gene (apo B cDNA nucleotides 8729 9854) was enzymatically amplified using Thermus aquaticus DNA polymerase (21), oligonucleotides B67-1 (5' AAATACACTGGAtCcTAGTAATGGAGTGATTG 3', apo B cDNA nucleotides 8720-8751) and B67-2 (5' CTGAAAAATCTCACGACGAGCTCCCCAGGACC 3 ', complementary to apo B cDNA nucleotides 9832-9863), and $0.5 \mu \mathrm{g}$ of genomic DNA prepared from peripheral white blood cells. Two base mismatches (lower-case letters above) were included in B67-1 to create a BamHI site. Enzymatic amplification was performed for $\mathbf{4 0}$ cycles, with denaturation, annealing, and extension temperatures of $96^{\circ} \mathrm{C}$, $65^{\circ} \mathrm{C}$, and $72^{\circ} \mathrm{C}$, respectively. The amplified DNA from subject II-2 was purified from a polyacrylamide gel and digested with $\mathrm{BamHI}$ and $E c o$ RI, and then a fragment containing apo B cDNA nucleotides 87299607 was cloned into M13 for DNA sequencing. DNA sequencing was performed by the dideoxynucleotide chain termination technique of Sanger et al. (22).

After the mutation responsible for apo B67 had been determined, the presence of this mutation in other family members was assessed by analyzing freshly prepared lipoprotein samples for the presence of apo B67 by SDS-PAGE.

\section{Results}

A pedigree illustrating the apo B67 kindred is shown in Fig. 1. The proband (subject I-1) had total and LDL cholesterol levels of 151 and $47 \mathrm{mg} / \mathrm{dl}$, respectively (Table I). These cholesterol values fall below the 5 th percentile for population controls. His apo B level was $48 \mathrm{mg} / \mathrm{dl}$, again below the 5 th percentile. The results of thyroid tests, as well as vitamin $A$ and $E$ levels, were normal. The HDL cholesterol and apo A-I levels were $103 \mathrm{mg} /$ $\mathrm{dl}$ (Table I) and $212 \mathrm{mg} / \mathrm{dl}$ (not shown), values well above the 95th percentile for population controls. An examination of the proband's VLDL revealed a unique truncated species of apo $B$, apo B67, in addition to the normal apo B species, apo B100 and apo B48 (Fig. 2). The apo B67 was also easily detectable in the IDL and LDL density iractions. No apo B67 was observed in the HDL fraction by Coomassie Blue-stained gels (Fig. 2); however, small amounts of apo B67 were detectable in the HDL fraction on silver-stained gels (not shown). The apo B67-containing particles within the LDL density range were easily demonstrable by nondenaturing gradient polyacrylamide gel electrophoresis (Fig. 3). The particles were smaller than normal

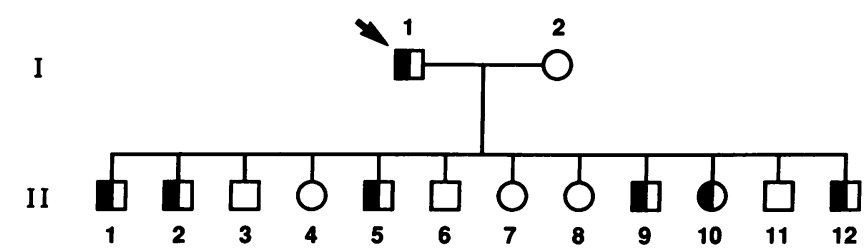

Figure 1. Pedigree showing the proband of the apo B67 kindred, his spouse, and 12 of his offspring. The proband, subject I-1, is indicated by the arrow. Subjects possessing the apo B67 allele are indicated by a half-shaded circle (female) or square (male). I and II denote the two generations of the proband's family. The concentrations of the lipoproteins in the plasma for each subject illustrated on this pedigree are listed in Table I. Lipoprotein profiles on five siblings of the proband indicated that none of the proband's siblings had hypobetalipoproteinemia. The proband actually had 14 offspring; however, one was deceased and one other refused to be interviewed or have his blood sampled. Those two family members are not shown on this pedigree. 
Table I. Lipoprotein Concentrations in the Apolipoprotein B67 Kindred

\begin{tabular}{|c|c|c|c|c|c|}
\hline Subject no. & Age/Sex & $\begin{array}{l}\text { Total plasma } \\
\text { cholesterol }\end{array}$ & $\begin{array}{c}\text { HDL } \\
\text { cholesterol }\end{array}$ & $\begin{array}{c}\text { LDL } \\
\text { cholesterol* }^{*}\end{array}$ & Triglycerides \\
\hline & $y r$ & & & & \\
\hline \multicolumn{6}{|c|}{ Apo B 67 heterozygotes } \\
\hline $\mathrm{I}-1$ & $81 / \mathrm{M}$ & 151 & 103 (>95th) & 47 (<5th) & $6(<5$ th $)$ \\
\hline II-1 & $55 / \mathrm{M}$ & 114 & 77 (>95th) & $33(<5$ th $)$ & 19 (<5th) \\
\hline II-2 & $54 / \mathrm{M}$ & 138 & 73 (>95th) & 64 (<5th) & $6(<5$ th $)$ \\
\hline II-5 & $48 / \mathrm{M}$ & 122 & 63 ( 95th) & $56(<5$ th $)$ & $16(<5$ th $)$ \\
\hline II-9 & $40 / \mathrm{M}$ & 110 & 64 (>95th) & $38(<5$ th $)$ & $16(<5$ th $)$ \\
\hline II- 10 & $38 / \mathrm{F}$ & 113 & 69 ( 85th) & $40(<5$ th $)$ & 20 (<5th) \\
\hline II -12 & $33 / \mathrm{M}$ & 95 & 75 (>95th) & $18(<5$ th $)$ & $10(<5$ th $)$ \\
\hline Mean \pm SD & & $120 \pm 19$ & $75 \pm 13$ & $42 \pm 15$ & $13 \pm 6$ \\
\hline \multicolumn{6}{|c|}{ Unaffected subjects ${ }^{\ddagger}$} \\
\hline $\mathrm{I}-2$ & $81 / \mathrm{F}$ & 278 & 63 ( 50th) & 196 (>90th) & $96(\sim 25$ th \\
\hline II-3 & $52 / \mathrm{M}$ & 187 & 38 ( 25th) & $126(\sim 35$ th $)$ & $114(\sim 50$ th \\
\hline II-4 & $50 / \mathrm{F}$ & 253 & 61 ( 50th) & $176(\sim 85$ th $)$ & $80(\sim 25$ th \\
\hline II-6 & $45 / \mathrm{M}$ & 159 & 48 ( 50th) & 101 ( 10th) & 48 (<5th) \\
\hline II-7 & $43 / F$ & 184 & $55(\sim 50$ th $)$ & $117(\sim 25$ th $)$ & $63(\sim 25$ th \\
\hline II-8 & $41 / \mathrm{F}$ & 184 & 64 ( 75th) & 108 ( 25th) & $60(\sim 10$ th \\
\hline II-11 & $36 / \mathrm{M}$ & 173 & 57 ( 85th) & $100(\sim 20$ th $)$ & $79(\sim 25$ th \\
\hline Mean \pm SD & & $203 \pm 45$ & $55 \pm 9$ & $132 \pm 38$ & $77 \pm 32$ \\
\hline$P$ value & & 0.001 & 0.01 & 0.001 & 0.001 \\
\hline
\end{tabular}

* The LDL cholesterol concentration percentile according to age- and sex-matched controls in the Lipid Research Clinics Prevalence Study (7) is shown in parentheses. ${ }^{\ddagger}$ Family members whose lipoproteins did not contain apo B67.

LDL particles yet larger than the apo B37-containing particles found in the HDL fraction of subjects heterozygous for the apo B37 mutation.

Based on its migration on SDS-gels in relation to size standards, we predicted that apo B67 must contain 3,000-3,100 amino acids. Western blots using apo B-specific monoclonal antibodies with known epitopes (17) provided support for this estimate. Antibody MB44 (which binds between apo B residues 2488 and 2658 ) and antibody $4 \mathrm{G} 3$ (which binds between residues 2980 and 3084) bound to apo B67, whereas an antibody to a synthetic peptide containing apo B100 amino acids 3120 3159 and the carboxy-terminal monoclonal antibody MB47 (which binds between amino acids 3441 and 3569) did not bind

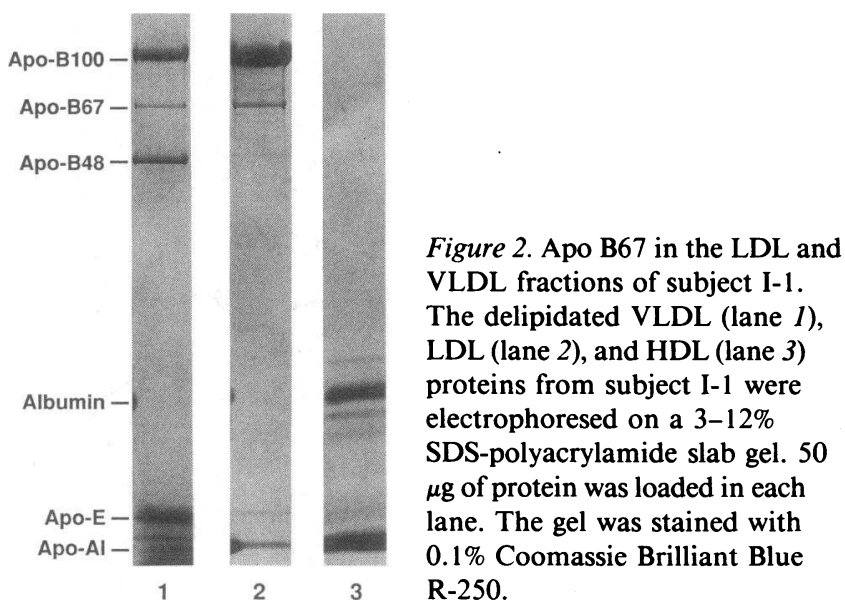

to apo B67 (Fig. 4). To determine the precise mutation accounting for apo B67, we enzymatically amplified the appropriate region of the apo B gene from the genomic DNA of subject II-2 and then subcloned it into M13 for sequencing. 4 of $10 \mathrm{M} 13$ clones contained a single nucleotide deletion in exon 26 of the apo B gene (cDNA nucleotide 9327). Because the DNA was cloned into mp18 as well as mp19, both DNA strands were sequenced, and the same deletion (Fig. 5) was identified in both strands. This deletion is predicted to yield a single novel amino acid followed by a premature stop codon (Fig. 5). The location of the mutation predicts that apo B67 contains 3040 amino acids.

The proband had 12 offspring. Neither the proband nor any of his children had clinical evidence of coronary artery disease, as judged by an electrocardiogram and the Rose questionnaire. 6 of the 12 children had hypobetalipoproteinemia, as defined by a fasting LDL cholesterol level below the 5 th percentile for age- and sex-matched controls (Table I). These six offspring were apo B67 heterozygotes, as determined by SDS-PAGE of the plasma lipoproteins. The triglyceride level in these seven subjects (the proband and the six offspring who were apo B67 heterozygotes) was remarkably low, averaging $13 \mathrm{mg} / \mathrm{dl}$. Interestingly, the HDL cholesterol levels in affected subjects averaged $75 \mathrm{mg} / \mathrm{dl}$, significantly higher than in unaffected family members $(P<0.01)$. All seven affected subjects had HDL cholesterols above the 85 th percentile for age- and sex-matched controls; six of the seven had HDL cholesterol levels at or above the 95 th percentile.

In every affected subject, the absolute concentration of apo B67 in plasma was low relative to that of the apo B100 produced by the normal allele. Based upon the relative staining 


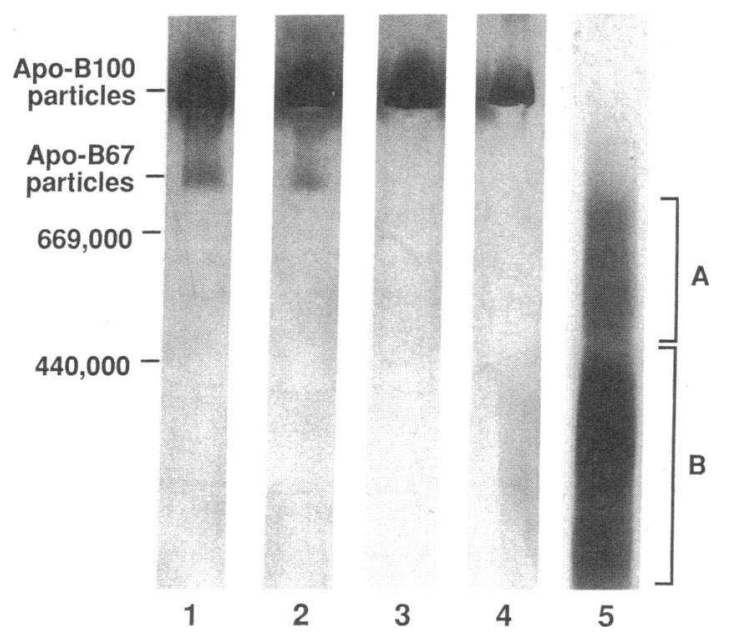

Figure 3. A nondenaturing, 2-16\% gradient polyacrylamide gel demonstrating the size of apo B67-containing LDL particles. A Pharmacia PAA 2/16 gel was used according to previously described methods $(15,19,20)$. Lanes 1 and 2 , LDL of two different hypolipoproteinemic family members (I-1 and II-2); lane 3 , the LDL of a normolipidemic control subject; lane 4, the LDL of an unaffected family member; lane 5, the HDL fraction of an apo B37 heterozygote (15). For each lane, 5-10 $\mu \mathrm{g}$ of protein was loaded; the gel was stained with silver. On Western blots, the band identified as apo B100 particles reacted with apo B-specific antibodies whose epitopes are in the amino-terminal and carboxy-terminal portions of apo B100 (data not shown). The band identified as apo B67 particles reacted only with antibodies whose epitopes are located between amino acids 1 and 3080, and not with the carboxy-terminal antibodies (data not shown). For lane 5, the migration of apo B37-containing particles within the HDL fraction is indicated by the bracket designated $A$; the migration of apo A-I-containing particles in the HDL fraction is indicated by the bracket designated $B$. The migration of the size standards is indicated: thyroglobulin $(669,000)$ and ferritin $(440,000)$.

intensities of the LDL apo B100 and apo B67 bands on SDSPAGE as well as the nondenaturing gels (Figs. 2 and 3), we have estimated that the plasma concentration of apo B67 is significantly $<5 \%$ of the apo B 100 concentration. The distribution of apo B67 within the different lipoprotein density classes in the

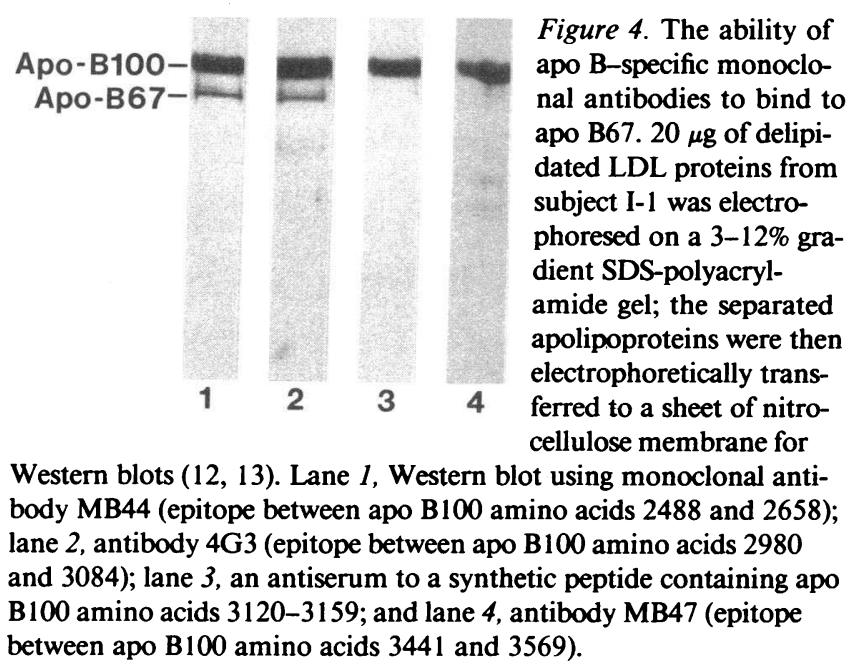

affected subjects in the second generation was identical to that observed in the proband (see above).

The mutation accounting for apo B67 occurs several kilobases $3^{\prime}$ to the edited nucleotide responsible for the synthesis of apo B48. One might therefore predict that the single nucleotide deletion in the apo B67 allele would not significantly affect apo B48 synthesis. If this were the case, apo B67 heterozygotes would have two alleles producing apo B48, yet only one making apo B100. Indirect support for this hypothesis was obtained by examining the VLDL of affected and unaffected individuals. When the VLDL of four affected subjects was analyzed by SDS-PAGE, we noticed that the amount of apo B48 in the VLDL, relative to apo $\mathrm{B} 100$, was significantly greater in the affected subjects (Fig. 6). We have recently observed the same increased apo B48/apo B100 ratio in the VLDL of apo B86 heterozygotes (23).

The dietary intake of affected and unaffected family members was determined from an analysis of a 3-d diet. No statistically significant differences in the total number of calories consumed or composition of the diet were observed (Table II). Similarly, we found no difference between affected and unaffected subjects in their activity level, as judged by the estimated number of miles walked per day. However, quite unexpectedly, we found a significant difference in their relative body weight and body mass index. The unaffected family members were significantly heavier than affected family members (Table II).

\section{Discussion}

In the past 3 years, reports by a number of investigators have made it clear that apo B gene mutations can cause familial hypobetalipoproteinemia (1). Each of the mutations reported to date has prevented the translation of a full-length apo B100. Some of these mutations have resulted in the production of a truncated form of apo B that can be identified within the plasma lipoproteins. In this paper, we report a unique apo B mutation associated with familial hypobetalipoproteinemia. A single nucleotide deletion in exon 26 of the apo $B$ gene causes the synthesis of a truncated species of apo B, apo B67. The apo B67 mutation provides important support for the hypothesis that the buoyant density of apo B-containing lipoproteins is inversely related to the length of the apo $B$ molecule. Previously, we have hypothesized that longer apo B species tend to form less dense (i.e., more buoyant) lipoproteins because longer forms of apo B would contain more lipid-binding regions $(1,24)$. The apo B67 mutation supports this hypothesis; apo B67-containing lipoproteins are, in general, more buoyant than apo B31- and apo B37-containing lipoproteins, yet slightly less buoyant than apo B86-containing lipoproteins. The density distribution of the various truncated apo B species that our laboratory has examined, including apo B67, is illustrated in Fig. 7.

The absolute concentration of the truncated apo B in plasma was low in affected family members, just as has been documented in other examples of truncated apo B's associated with hypobetalipoproteinemia (1). Whether the low levels of apo B67 are due to low synthetic rates or increased degradation rates is not yet known. Two longer truncated apo $B$ species, apo B87 and apo B89, have been reported to bind to the LDL 

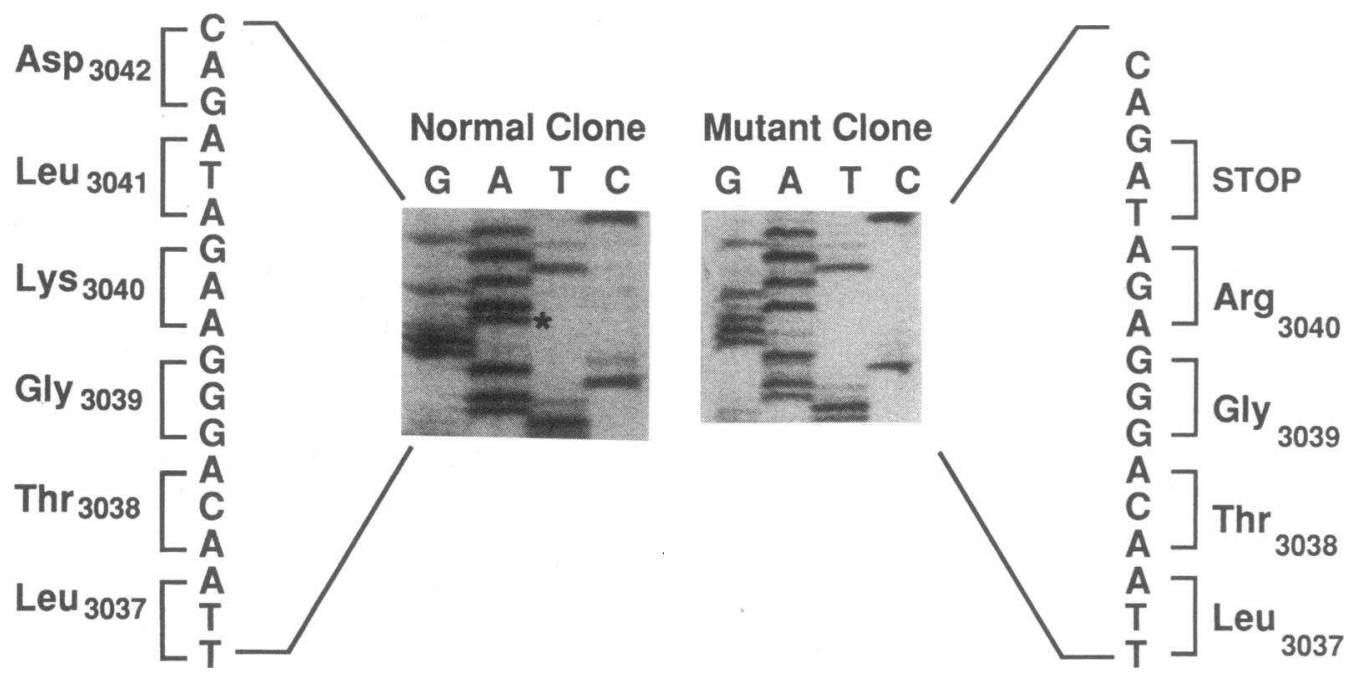

Figure 5. Autoradiograms of DNA sequencing gels demonstrating the mutation responsible for apo B67. The region of the apo $B$ gene suspected to contain the mutation was enzymatically amplified from the genomic DNA of subject II-2, and then an 879-base pair fragment spanning from the BamHI site created in oligonucleotide B67-1 to the EcoRI site at apo B cDNA nucleotide 9607 was subcloned into M13 for sequencing (see Methods). 6 of 10 subclones contained the normal apo B sequence (left), and four contained a deletion

of apo B cDNA nucleotide 9327 (right). The single nucleotide whose deletion defines the mutant allele is marked by an asterisk in the normal allele. This frameshift mutation is predicted to yield one novel amino acid, arginine, followed by a premature stop codon (TAG). No other changes from the consensus apo B sequence were noted in the mutant subclones.

receptor with enhanced affinity, possibly explaining the low levels of these truncated apo B species $(25,26)$. The ability of apo B67 to bind to the LDL receptor has not yet been experimentally tested. Apo B67 is predicted to contain 3,040 amino acids and contains the epitope for antibody $4 \mathrm{G} 3(16,17)$, an antibody widely used over the past 10 years for blocking the binding of LDL to the LDL receptor (Fig. 8). However, apo B67 terminates before epitopes for several other LDL receptorblocking antibodies, before the missense mutation at residue 3500 that is associated with defective binding of LDL to the LDL receptor, and before the two short positively charged amino acid sequences that have been hypothesized to be involved in receptor binding (Fig. 8). Because apo B67 does not contain these latter domains, it is our hypothesis that apo B67, like apo B37 (27), would not bind to the LDL receptor. We plan to purify apo B67-containing lipoproteins to test this possibility directly. The affected heterozygotes in this family have consented to lipoprotein turnover studies on the metabolic ward, and studies are being planned to search for the cause of the low levels of apo B67 in their plasma.

The significantly lower body weight in the affected subjects, compared with the unaffected subjects, was an unexpected finding. This result could be explained by postulating some degree of intestinal malabsorption. Counter to this hypothesis is the fact that the affected subjects were free of clinical symptoms of

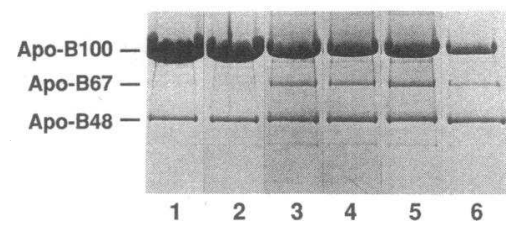

Figure 6. SDS-polyacrylamide gel of the VLDL fraction of normolipidemic control subjects and four different apo B67 heterozygotes. The VLDL population was isolated

from plasma obtained $2 \mathrm{~h}$ after a fat-rich meal. The delipidated VLDL proteins $(50 \mu \mathrm{g})$ were electrophoresed on a 3-12\% SDS-polyacrylamide slab gel, and the gel was stained with $0.1 \%$ Coomassie Blue. Lanes 1 and 2, VLDL from two different unaffected normolipidemic subjects; lanes 3-6, the VLDL from four different apo B67 heterozygotes. malabsorption; however, analyzing these subjects for subtle degrees of malabsorption would require sophisticated tests of fat absorption on the metabolic ward. Also, as noted in Results, one could make a persuasive argument that the apo B67 allele would yield normal amounts of apo B48 and that apo B67 heterozygotes would therefore synthesize completely normal amounts of apo B48 in the intestine. Some of our data demonstrate an increased amount of apo B48, relative to apo B100, in the VLDL of apo B67 heterozygotes (Fig. 6); these data are consistent with the hypothesis that apo B67 heterozygotes make normal amounts of apo B48, yet half-normal amounts of apo B100. Based on these arguments, we are skeptical about the possibility of intestinal malabsorption. It is possible that the significantly lower weight of affected subjects is the result of chance. This issue needs to be investigated further in other kindreds with hypobetalipoproteinemia.

Table II. Diet, Activity Load, and Weight of Apo B67 Heterozygotes and Unaffected Family Members

\begin{tabular}{lcc}
\hline & $\begin{array}{c}\text { Apo B67 } \\
\text { heterozygotes }\end{array}$ & $\begin{array}{c}\text { Unaffected } \\
\text { subjects }\end{array}$ \\
\hline Estimated calories consumed per day & $2,977 \pm 942$ & $2,614 \pm 813$ \\
Dietary composition* & & \\
$\quad$ Percent carbohydrate & $41 \pm 9.0$ & $43 \pm 9.3$ \\
Percent fat & $44 \pm 7.3$ & $41 \pm 7.5$ \\
Percent protein & $15 \pm 3.8$ & $16 \pm 3.8$ \\
Cholesterol (mg/day) & $665 \pm 317$ & $648 \pm 316$ \\
Subject weight/ideal body weight ${ }^{\ddagger}$ & $1.01 \pm 0.04$ & $1.20 \pm 0.18^{\S}$ \\
Body mass index $^{\ddagger}$ & $23.3 \pm 1.08$ & $28.6 \pm 2.88^{\prime \prime}$ \\
Estimated miles walked per day & $2.1 \pm 1.2$ & $2.8 \pm 1.4$ \\
\hline
\end{tabular}

Values shown in this table are means \pm standard deviation.

* Calculated from a 3-d dietary history using the University of Minnesota dietary analysis computer program (8).

$\ddagger$ Calculated according to method of Keys et al. (9).

${ }^{8} P=0.032 ; " ~ P=0.002$. 


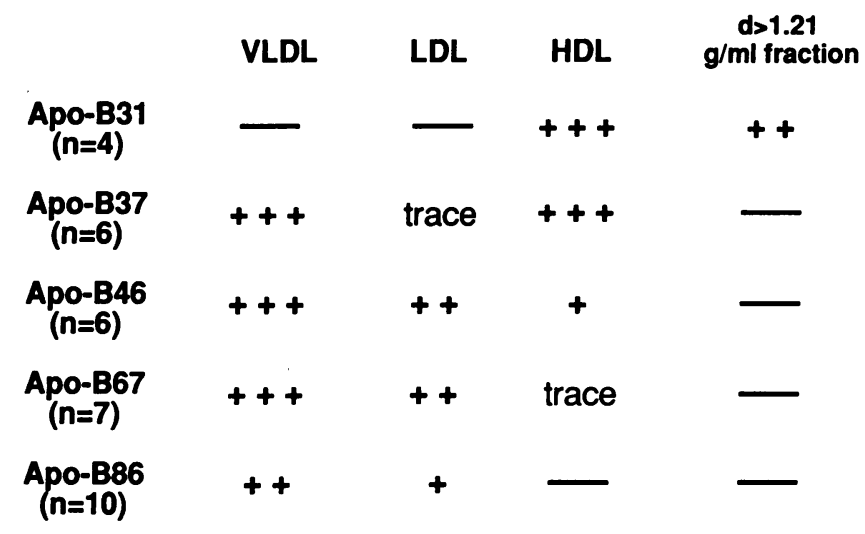

Figure 7. The distribution of truncated apo B species among the different lipoprotein density classes. The density ranges are as follows: VLDL, $d<1.006 \mathrm{~g} / \mathrm{ml}$; LDL, $d=1.025-1.063 \mathrm{~g} / \mathrm{ml}$; HDL, $d$ $=1.063-1.21 \mathrm{~g} / \mathrm{ml}$. The data shown here summarize the density distribution of apo B67, as well as that for other truncated species of apo B examined by our laboratory $(15,24,28,29)$. The distribution shown here is a subjective assessment of the distribution of each truncated apo B, based on the electrophoresis of each lipoprotein fraction on stained SDS-polyacrylamide gels.

An interesting aspect of this kindred is the fact that every affected subject had a high HDL cholesterol level. Six of the seven heterozygotes had an HDL cholesterol level at or exceeding the 95th percentile for age- and sex-matched controls. High HDL levels have previously been observed in all four apo B31 heterozygotes (24) and five of the six apo B46 heterozygotes (28), although the observation was not discussed in the description of those kindreds. However, high HDL cholesterol levels have not always been observed in the affected members of kindreds with hypobetalipoproteinemia heterozygotes (29). It is possible that another inherited trait or traits govern the ability to "respond" to low apo B levels with high HDL cholesterol levels.
The metabolic basis for the high HDL cholesterol levels in heterozygotes for hypobetalipoproteinemia requires further analysis. One possibility is that the paucity of apo B-containing lipoproteins in the plasma retards the cholesteryl ester transfer protein-mediated transfer of HDL cholesterol to more buoyant lipoproteins. If this were the mechanism for the high HDL cholesterol levels, one might expect to find an accumulation of $\mathrm{HDL}_{2}$ in their plasma, as occurs in a deficiency of cholesterol ester transfer protein, where there is a defect in the transfer of $\mathrm{HDL}_{2}$ cholesterol to apo B-containing lipoproteins (30). However, in preliminary studies, we have found elevated $\mathrm{HDL}_{3}$ and normal $\mathrm{HDL}_{2}$ levels in two apo B67 heterozygotes, as determined by dextran sulfate precipitation. Both subject I-1 and subject II-2 had normal $\mathrm{HDL}_{2}$ levels, but significantly elevated $\mathrm{HDL}_{3}$ levels. The apo A-I, but not the apo A-II, levels were elevated in these two subjects (F. Welty and M. Cheung, unpublished observations). The same pattern-a disproportionate amount of $\mathrm{HDL}_{3}$-has been reported in other subjects with well-documented cases of hypobetalipoproteinemia $(15,31)$. Another possibility is that the high HDL cholesterol levels might be a consequence of altered metabolism of triglyceriderich lipoproteins. Hypoalphalipoproteinemia is frequently observed in subjects with hypertriglyceridemia. Our affected subjects had hyperalphalipoproteinemia associated with extraordinarily low plasma triglyceride levels. Recently, Ginsberg and co-workers (32) have reported that normotriglyceridemic subjects with hypoalphalipoproteinemia tend to have a high apo A-I fractional catabolic rate as well as a high LDL-apo B production rate. They suggested the possibility that a portion of the increased heart disease risk in subjects with hypoalphalipoproteinemia is due to elevated LDL-apo B production rate (32). We suggest that hypobetalipoproteinemia may represent the other end of the spectrum from the subjects characterized by Ginsberg and co-workers (32). Perhaps heterozygotes for hypobetalipoproteinemia, whose LDL-apo B production rates are almost certainly very low (33), have a decreased catabolic rate for apo A-I. We plan to test this hypothesis by performing apo B100 and apo A-I turnover studies in affected subjects.

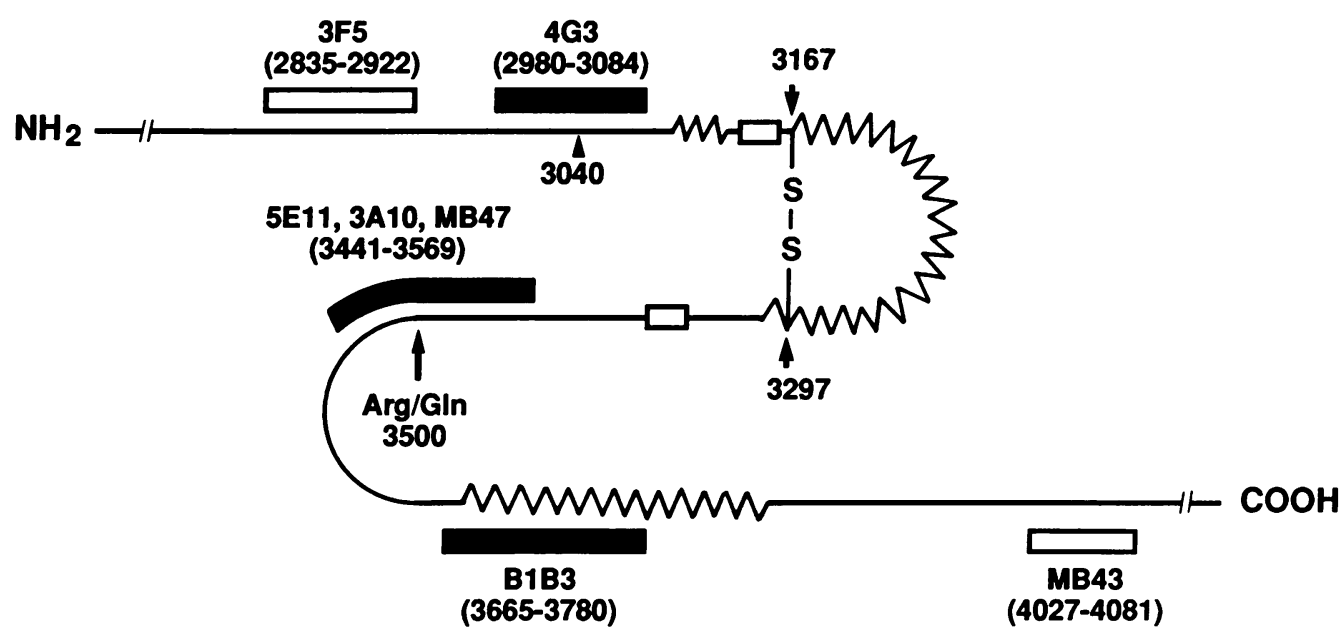

Figure 8 . The location of the carboxy-terminal amino acid of apo B67 (amino acid 3040) in relation to the receptor-binding domain of apo B. The epitopes of monoclonal antibodies that totally or partially block binding of LDL to the LDL receptor are indicated by solid and open boxes, respectively, above or below the polypeptide chain. Apo B67 contains the epitope for antibody 4G3 (see Fig. 4). The two short stretches of positively charged amino acids are indicated by open boxes within the polypeptide chain; zigzag lines indicate regions of potential amphipathic $\beta$-sheet structure. The location of the Arg $\rightarrow$ Gln substitution associated with familial defective apo B100 is indicated. (Modified, with permission, from Milne, R., et al. 1989. J. Biol. Chem. 264:19754-19760.) 


\section{Acknowledgments}

We thank Drs. J. Witztum and L. Curtiss of the La Jolla Arteriosclerosis SCOR program for apo B-specific monoclonal antibodies; Drs. $\mathbf{R}$. Milne and Y. Marcel for the apo B-specific monoclonal antibody 4G3; Dr. T. Innerarity for the antiserum to the apo B synthetic peptide 3120 3159; Drs. J. and C. Seidman for help and encouragement; M. Cheung for analyzing the HDL subfractions; J. Miller, B. Knott, and M. Kowalchuk for technical support; $T$. Gridley for manuscript preparation; $T$. Rolain and C. Benedict for graphics; and A. Averbach and S. G. Seehafer for editorial assistance.

Dr. Welty is a Teaching and Research Scholar of the American College of Physicians. This research was supported by grants from the National Institutes of Health (HL-0162 and HL-41633), a Syntex Scholars Award (Dr. Young), and the Medical Center of Delaware, Wilmington, DE (Dr. Welty).

\section{References}

1. Young, S. G. 1990. Recent progress in understanding apolipoprotein B. Circulation. 82:1574-1594.

2. Malloy, M. J., and J. P. Kane. 1982. Hypolipidemia. Med. Clin. North Am. 66:469-484.

3. Kahn, J. A., and C. J. Glueck. 1978. Familial hypobetalipoproteinemia: absence of atherosclerosis in a postmortem study. JAMA (J. Am. Med. Assoc.). 240:47-48.

4. Glueck, C. J., P. S. Gartside, M. J. Mellies, and P. M. Steiner. 1977. Familial hypobeta-lipoproteinemia: studies in 13 kindreds. Trans. Assoc. Am. Physicians. 90:184-203.

5. Welty, F. K. 1989. Cosegregation of hypobeta- and hyperalpha-lipoproteinemia in a kindred. Circulation. 80:II-276.

6. LaCroix, A. Z., J. M. Guralnik, J. D. Curb, R. B. Wallace, A. M. Ostfeld, and C. H. Hennekens. 1990. Chest pain and coronary heart disease mortality among older men and women in three communities. Circulation. 81:437-446.

7. Lipid Research Clinics Program. 1974. Manual of Laboratory Operations, Volume 1. Lipid and Lipoprotein Analysis. DHEW Publication No. 75-628 (NIH), 2nd edition. Government Printing Office, Washington, DC.

8. Schakel, S. F., Y. A. Sievert, and I. M. Buzzard. 1988. Sources of data for developing and maintaining a nutrient database. Dietary Intake Methods. 88:1268-1271.

9. Keys, A., F. Fidanza, M. J. Karvonen, N. Kimura, and H. L. Taylor. 1972. Indices of relative weight and obesity. J. Chron. Dis. 25:329-343.

10. Havel, R. J., H. A. Eder, and J. H. Bragdon. 1955. The distribution and chemical composition of ultracentrifugally separated lipoproteins in human serum. J. Clin. Invest. 34:1345-1353.

II. Lowry, O. H. N. J. Rosebrough, A. L. Farr, and R. J. Randall, 1951. Protein measurement with the Folin phenol reagent. J. Biol. Chem. 193:265-275.

12. Curtiss, L. K., and T. S. Edgington. 1982. Immunochemical heterogeneity of human plasma apolipoprotein B. I. Apolipoprotein B binding of mouse hybridoma antibodies. J. Biol. Chem. 257:15213-15221.

13. Young, S. G., S. J. Bertics, T. M. Scott, B. W. Dubois, L. K. Curtiss, and J. L. Witztum. 1986. Parallel expression of the MB19 genetic polymorphism in apoprotein B-100 and apoprotein B-48: evidence that both apoproteins are products of the same gene. J. Biol. Chem. 261:2995-2998.

14. Young, S. G., J. L. Witztum, D. C. Casal, L. K. Curtiss, and S. Bernstein. 1986. Conservation of the low density lipoprotein receptor-binding domain of apoprotein B: demonstration by a new monoclonal antibody, MB47. Arteriosclerosis. 6:178-188.

15. Young, S. G., S. J. Bertics, L. K. Curtiss, and J. L. Witztum. 1987. Characterization of an abnormal species of apolipoprotein B, apolipoprotein B-37, associated with familial hypobetalipoproteinemia. J. Clin. Invest. 79:1831-1841.

16. Milne, R. W., R. Theolis Jr., R. B. Verdery, and Y. L. Marcel. 1983.
Characterization of monoclonal antibodies against human low density lipoprotein. Arteriosclerosis. 3:23-30.

17. Milne, R., R. Théolis Jr., R. Maurice, R. J. Pease, P. K. Weech, E. Rassart, J. C. Fruchart, J. Scott, and Y. L. Marcel. 1989. The use of monoclonal antibodies to localize the low density lipoprotein receptor-binding domain of apolipoprotein B. J. Biol. Chem. 264:19754-19760.

18. Innerarity, T. L., S. G. Young, K. S. Poksay, R. W. Mahley, R. S. Smith, R. W. Milne, Y. L. Marcel, and K. H. Weisgraber. 1987. Structural relationship of human apolipoprotein B48 to apolipoprotein B100. J. Clin. Invest. 80:1794 1798.

19. Curtiss, L. K., and T.S. Edgington. 1985. Immunochemical heterogeneity of human plasma high density lipoproteins: identification with apolipoprotein A-I- and A-II-specific monoclonal antibodies. J. Biol. Chem. 260:2982-2993.

20. Blanche, P. J., E. L. Gong, T. M. Forte, and A. V. Nichols. 1981. Characterization of human high-density lipoproteins by gradient gel electrophoresis. Biochim. Biophys. Acta. 665:408-419.

21. Saiki, R. K., D. H. Gelfand, S. Stoffel, S. J. Scharf, R. Higuchi, G. T. Horn, K. B. Mullis, and H. A. Erlich. 1988. Primer-directed enzymatic amplification of DNA with a thermostable DNA polymerase. Science (Wash. DC). 239:487-491.

22. Sanger, F., S. Nicklen, and A. R. Coulson. 1977. DNA sequencing with chain-terminating inhibitors. Proc. Natl. Acad. Sci. USA. 74:5463-5467.

23. Linton, M. F., V. R. Plerotti, S. T. Hubl, and S. G. Young. 1990. An apo-B gene mutation causing familial hypobetalipoproteinemia analyzed by examining the apo-B cDNA amplified from the fibroblast RNA of an affected subject. Clin. Res. 38:286A. (Abstr.)

24. Young S. G., S. T. Hubl, R. S. Smith, S. M. Snyder, and J. F. Terdiman 1990. Familial hypobetalipoproteinemia caused by a mutation in the apolipoprotein B gene that results in a truncated species of apolipoprotein B (B-31): a unique mutation that helps to define the portion of the apolipoprotein B molecule required for the formation of buoyant, triglyceride-rich lipoproteins. J. Clin. Invest. 85:933-942.

25. Krul, E. S., M. Kinoshita, P. Talmud, S. E. Humphries, S. Turner, A. C Goldberg, K. Cook, E. Boerwinkle, and G. Schonfeld. 1989. Two distinct truncated apolipoprotein B species in a kindred with hypobetalipoproteinemia. Arteriosclerosis. 9:856-868.

26. Gabelli, C., G. Baggio, C. Bilato, S. Martini, L. Previato, Y. Marcel, A. Corsini, and G. Crepaldi. 1989. Identification of a new apoB variant associated with hypobetalipoproteinemia. Circulation. 80(Suppl. II):II-276.

27. Young, S. G., F. P. Peralta B. W. Dubois, L. K. Curtiss, J. K. Boyles, and J. L. Witztum. 1987. Lipoprotein B37, a naturally occurring lipoprotein containing the amino-terminal portion of apolipoprotein B100, does not bind to the apolipoprotein B,E (low density lipoprotein) receptor. J. Biol. Chem. 262:16604 16611.

28. Young, S. G., S. T. Hubl, D. A. Chappell, R. S. Smith, F. Claiborne, S. M. Snyder, and J. F. Terdiman. 1989. Familial hypobetalipoproteinemia associated with a mutant species of apolipoprotein B (B46). N. Engl. J. Med. 320:1604 1610 .

29. Young, S. G., S. J. Bertics, L. K. Curtiss, B. W. Dubois, and J. L. Witztum. 1987. Genetic analysis of a kindred with familial hypobetalipoproteinemia. Evidence for two separate gene defects: one associated with an abnormal apolipoprotein B species, apolipoprotein B-37; and a second associated with low plasma concentrations of apolipoprotein B-100. J. Clin. Invest. 79:1842-1851.

30. Brown, M. L., A. Inazu, C. B. Hesler, L. B. Angellon, C. Mann, M. E. Whitlock, Y. L. Marcel, R. W. Milne, J. Kolzumi, H. Mabuchi, et al. 1989. Molecular basis of lipid transfer protein deficiency in a family with increased high-density lipoproteins. Nature (Lond.). 342:448-451.

31. Malloy, M. J., J. P. Kane, D. A. Hardman, R. L. Hamilton, and K. B. Dalal. 1981. Normotriglyceridemic abetalipoproteinemia: absence of the B-100 apolipoprotein. J. Clin. Invest. 67:1441-1450.

32. Ginsberg, H. N., C. Ngai, and R. Ramakrishnan. 1990. Increased low density lipoprotein production rates in subjects with isolated reductions in high density lipoprotein cholesterol levels. Clin. Res. 38:482A. (Abstr.)

33. Sigurdsson, G., A. Nicoll, and B. Lewis. 1977. Turnover of apolipoprotein-B in two subjects with familial hypobetalipoproteinemia. Metab. Clin. Exp. 26:25-30. 